\title{
The dependence of semantic relatedness effects upon prime processing
}

\author{
AVISHAI HENIK \\ Ben-Gurion University of the Negev, Beer Sheva, Israel \\ FRANCES J. FRIEDRICH \\ Good Samaritan Hospital and Medical Center, Portland, Oregon \\ and \\ WENDY A. KELLOGG \\ University of Oregon, Eugene, Oregon
}

\begin{abstract}
The semantic relationship between a prime and a target word has been shown to affect the speed at which the target word is processed. This series of experiments investigated how the semantic priming effect is influenced by the nature of the task performed on the prime word. Subjects were asked to perform either a naming or a letter-search task on the prime word and either a lexical-decision or color-naming task on the target word. When the primes were named, response times for the target words were facilitated in the lexical-decision task and inhibited in the color-naming task. However, these effects were eliminated or reduced to an insignificant level when the primes were searched for letters. We suggest that in order to produce the usual priming effect, the primes have to be processed for meaning rather than probed for constituents.
\end{abstract}

The past decade of experimentation in cognitive psy. chology has been marked by an effort to reveal the nature of semantic memory structures that are assumed to underlie mental processing. A major technique used to study the basic characteristics of the semantic memory network is the priming paradigm. In this paradigm, the subject is presented with two successive stimuli called the prime and the target, respectively. The subject is asked to respond overtly only to the target. It is by now well documented that semantic relatedness between the prime and the target facilitates the processing of the target in a variety of situations (e.g., Meyer \& Schvaneveldt, 1971, 1976; Schvaneveldt \& McDonald, 1981 ; Smith, 1979).

A common assumption among researchers in the field is that the stimulation of a concept (a node in the semantic network) causes a spread of activation to related concepts in the memory system (Collins \& Loftus,

This research was supported in part by Grants $80-10-8$ from the Sloan Foundation and BNS 7923527.02 from the National Science Foundation to Michael Posner at the University of Oregon. The third author was supported by a National Science Foundation Predoctoral Fellowship. We gratefully acknowledge the helpful comments provided by Michael Posner at all stages of this research. We also thank Kathy Hollosy for her help in preparation of the manuscript. This research was conducted while the first two authors carried out postdoctoral work at the University of Oregon. Requests for reprints should be addressed to the second author at the Cognitive Neuropsychology Laboratory, Suite 280, 2222 N.W. Lovejoy, Portland, Oregon 97210.
1975; Posner \& Snyder, 1975a, 1975b). Thus, the semantic-relatedness effect can be explained as a larger or more reliable spread of activation for concepts that are semantically related than for those that are not. Moreover, it seems that this spread of activation is automatic in that it is not dependent upon conscious attention or the subjects' intentions (Marcel, 1980; Neely, 1977).

In addition, several authors have discussed the role of attentional (Neely, 1976, 1977; Posner \& Snyder, 1975a, 1975b; Tweedy \& Lapinski, 1981) and strategic (Becker, 1980) factors in producing semantic priming effects. Factors such as the proportion of related word pairs or the consistency in strength of the semantic relationship appear to influence the expectations of the subjects and affect the resulting patterns of facilitation and inhibition. Neely (1977) demonstrated that both automatic spreading activation and conscious attentional processes underlie semantic priming effects and that the time courses of the two processes can be charted separately.

An important aspect of semantic priming effects that has received little attention revolves around the mode of processing the prime word. In general, the subject is asked to look at the prime without making a response of any kind; thus, the mode of processing the prime is not controlled. It is possible that spreading activation, as measured by a semantic priming effect, will occur regardless of the nature of the task performed on the prime word. Alternatively, it is possible that the mode of 
processing adopted by the subject will constrain automatic activation of semantically related elements in the semantic network.

Evidence that the nature of the processing of a priming word can alter the normal pattern of semantic priming was found in a series of experiments by Smith (1979). In the first experiment, Smith showed that semantic priming could be obtained with a letter-search task on target words. Subjects were presented with a prime word and then with a target word with a probe letter above it and were asked to search for the probe letter in the target. The subjects' reaction times (RTs) were faster when the target and the prime were identical or semantically related than when they were unrelated. In Experiments 2 and 3, the subjects were asked to search for a letter in the prime as well as in the target. This manipulation eliminated the relatedness effect. Smith suggested that contextual facilitation in the priming paradigm depends upon the mode of analysis applied to the prime. Facilitation occurs when the prime is analyzed as a whole for its meaning. However. if the focus of prime processing is on its constituents, the effect disappears. This is an important result, because it suggests that there may be constraints on the automatic activation of the prime or the subsequent spread of activation.

Unfortunately, Smith's (1979) results are not conclusive with regard to this issue. As she pointed out, the lack of facilitation obtained when both the prime and the target were searched for letters may have been due to the task demands. One possibility is that the letter search on the target is performed differently when there is also a letter search on the prime. Performing a letter search may normally involve lexical access (see Smith, 1979), although this is not a logical necessity. Lexical access in the letter-search task is supported, however, by the fact that a semantic priming effect can be obtained when a letter search is performed on the target alone (Smith, 1979) and the finding that words are searched for letters more rapidly than are pronounceable nonwords (Krueger \& Weiss, 1976). Thus, one might expect faster letter-search decisions when lexical access occurs than when the search is performed solely at a lower level of analysis. If a prior letter search on the prime induces a shift in target processing to a prelexical level, then any activation in the semantic network produced by the prime might be precluded from having a facilitatory effect on target RTs. Smith's results showed that RTs to targets were much slower (by about $200 \mathrm{msec}$ ) when a letter search was performed on both the prime and the target, even with a relatively long stimulus onset asynchrony $(1700 \mathrm{msec})$. The long RTs may thus reflect the time to perform the letter search on the target without the aid of lexical activation.

Another explanation for the lack of facilitation when both primes and targets are searched is that there may be a special difficulty in searching the same word successively for different letters. As Smith (1979) pointed out, such a specific inhibitory effect could account for the loss of facilitation when primes and targets were identical but might not explain the lack of facilitation in the semantically related pairs. However, it is interesting to note that although no benefit in target RT for semantically related pairs was observed, a significant $(p<.01)$ benefit in errors was observed. It is thus possible that automatic lexical activation of the prime did occur when it was searched for a letter, but that other experimental factors obscured a priming effect in the RT measure. We carried out a series of experiments that addressed this issue directly by manipulating the task requirements for the prime and by using tasks on the target that involved lexical activation.

\section{EXPERIMENT 1}

In the first experiment, a lexical decision was made on the target string, and a letter search was performed on the prime. The lexical-decision task necessitates lexical access of the target and thus rules out the possibility mentioned by Smith (1979) that performing a letter search on the prime may induce a shift in the mode of processing the target that precludes contextual facilitation. To ensure that the usual priming effect would occur in our task, on half of the trials (separate blocks) we asked the subjects to name the prime rather than to search it for a letter. The subjects performed a lexical decision task on the targets throughout the experiment.

\section{Method}

Stimuli and Apparatus. Each trial consisted of a prime word and a target string of letters. Priming stimuli were always a single word with a probe letter above. In order to avoid any probeposition effects, the probe letter was presented above every letter in the prime: for example,

\section{EEEEE}

UNDER

On half of the trials, the probe represented a letter in the prime word; on the other half, it did not. For positive trials, the position of the probe letter was drawn equally often from the beginning, the middle, and the end of the word. There were three kinds of stimulus pairs: related words, unrelated words, and word-nonword pairs. The related pairs were chosen from the Keppel and Strand (1970) and Postman (1970) norms. The pairs consisted of the stimulus and its strongest associate. Each word in each pair was used three times in order to generate the three different stimulus pairs. The set of pronounceable nonwords was created by replacing a letter in each of the target words. Nonwords were always paired with a prime word that was unrelated to the target from which the nonword was derived.

The stimuli were presented on a television screen that was at eyc level, approximately $60 \mathrm{~cm}$ from the subject. On this screen, each letter was $1 \mathrm{~cm}$ high and $.8 \mathrm{~cm}$ wide, and the center-tocenter distance between adjacent letters was $1 \mathrm{~cm}$. Vocal responses were recorded with a voice-operated relay (GrasonStadler Model E7200A-1), which was interfaced with an Apple II computer. Stimulus presentations and data recording were controlled by the Apple II computer.

Design. There were 150 experimental trials. One third of them were related, one third unrelated, and one third word- 
nonword pairs. Half (25) of the trials in each of these groups included positive letter-search trials, and half included negative letter-search trials. Practice consisted of an additional 48 trials that were designed in the same way as the experimental trials. Each series of trials (practice and experimental) was split into three blocks. The allocation of trials to blocks was random and varied from subject to subject. These 198 trials were repeated twice in a session, once for each prime task. The order of the prime task was varied such that half of the subjects performed the letter search first and the other half performed the naming task first.

Procedure. Each subject participated in a 1-h session. Practice trials were given in order to familiarize the subjects with the tasks. Each block of trials was preceded by the word "READY." A keypress by the subject initiated the block. The prime appeared for $1 \mathrm{sec}$ and was followed by a blank interval of $700 \mathrm{msec}$ prior to the target presentation. The target exposure was terminated by the subject's keypress.

The subjects were asked to respond vocally to the prime and manually to the target. The latency of the vocal response was monitored by the voice-operated relay. When the prime was searched for a letter, the subjects responded "yes" or "no" and the response was typed into the computer by the experimenter When the subjects named the prime, the experimenter recorded whether the response was correct or incorrect. Throughout the experiment, the subjects performed a lexical decision on the targets and indicated their decisions by pressing one of two keys. Whenever an error in the lexical-decision task was made, the word "INCORRECT" appeared on the screen for $2 \mathrm{sec}$. No feedback was given for the letter-search task.

Subjects. Fourteen University of Oregon students were recruited from the subject pool of the Cognitive Laboratory. They were paid $\$ 3.00$ for their participation. All subjects were native English speakers.

\section{Results and Discussion}

Response latencies and error rates for the lexical decisions were analyzed. Error rates were quite low. When the primes were searched for letters, the error rates were $2.29 \%$ in the unrelated condition, compared with $1.43 \%$ for the related pairs. When the primes were named, the error rates were $1.14 \%$ and $1.13 \%$ for unrelated and related pairs, respectively.

For each subject, a median RT was computed for the correct trials in each condition. A trial was defined as correct when the responses to both the prime and target were correct. These data were subjected to a three-way repeated-measures analysis of variance, with prime task (letter search or naming) and relatedness (related or unrelated pairs) as within-subjects variables and order (letter search first or letter search second) as a betweensubjects variable.

Collapsed over all other variables, lexical decisions were $17 \mathrm{msec}$ faster in the related than in the unrelated pairs $[F(1,12)=12.99, p<.01]$ and were $31 \mathrm{msec}$ faster when the prime task was naming than when it was a letter search $[F(1,12)=5.59, p<.05]$. However, these two factors also produced a significant interaction $[F(1,12)=9.97, p<.01]$. These data are presented in Table 1 . When the prime was named, the average lexicaldecision time for the targets was $34 \mathrm{msec}$ faster for the related pairs than for the unrelated pairs. However, when the prime was searched for a letter, there was no effect of relatedness. An analysis of the data of individual subjects revealed that in the letter-search task, 8
Table 1

Mean Reaction Times (in Milliseconds) of Lexical Decision as a Function of Relatedness and Prime Task

\begin{tabular}{ccc} 
& \multicolumn{2}{c}{ Prime Task } \\
\cline { 3 - 3 } Relatedness & Letter Search & Naming \\
\hline Related & 580 & 532 \\
Unrelated & 580 & 566 \\
\hline
\end{tabular}

subjects showed a relatedness effect and 6 did not. When the prime was named, 13 showed the effect and 1 did not $(\mathrm{p}<.001$ by the sign test).

Task order interacted significantly with prime task $[F(1,12)=6.18, p<.05]$ and relatedness $[F(1,12)=$ $8.89, \mathrm{p}<.025]$. When the prime letter-search task was performed in the first half of the session and the prime naming task in the second, lexical decisions (collapsed across relatedness) were $63 \mathrm{msec}$ faster in the naming task than in the letter-search task; when task order was reversed, lexical-decision times in the prime naming task were $2 \mathrm{msec}$ slower. Similarly, the overall effect of relatedness was $32 \mathrm{msec}$ when the prime letter-search task was first, compared with $3 \mathrm{msec}$ when the task order was reversed. In general, it appears that prime task and relatedness effects were attenuated when the prime letter-search task occurred second. However, it is important to note that the three-way interaction among prime task, relatedness, and order did not approach significance. That is, the interaction of prime task and relatedness, discussed above, did not depend on the order in which the subjects performed the tasks.

The results of Experiment 1 suggest that the usual semantic priming effect is eliminated when a letter search is performed on the prime, even with a target task that requires lexical activation. However, before drawing any firm conclusions, we attempted to replicate the lack of facilitation in Experiment 2, in which the subjects performed the prime letter-search task in both halves of the session. Having the same subjects perform the letter-search task twice allowed examination of the effect of the repetition of stimulus materials, as well as assessment of any confounding influence of set that may have resulted from task order in Experiment 1. If the semantic priming effect was dependent on the type of task performed on the prime, the lack of a relatedness effect should persist in Experiment 2. If, however, the lack of a priming effect in the letter-search condition of Experiment 1 resulted from practice with the stimulus materials or a specific influence of performing the prime naming task first, semantic facilitation might be observed in the first half of the session, leading to a relatedness $x$ practice interaction.

\section{EXPERIMENT 2}

\section{Method}

In this experiment, only the prime letter-search task was used. The experiment started with 48 practice trials, followed by 
two administrations of experimental trials. In all other respects, the experiment was the same as the prime letter-search condition of the previous experiment.

Subjects. Ten University of Oregon students were recruited from the subject pool of the Cognitive Laboratory. They were paid \$3.00 for their participation. All subjects were native English speakers, none of whom had participated in Experiment 1 .

\section{Results}

Response latencies and error rates for the lexical decisions were analyzed. In the first half of the session, there were $0.40 \%$ errors in the related trials and $0.12 \%$ errors in the unrelated trials. In the second half, the error rate was $0.80 \%$ for both related and unrelated trials.

For each subject, a median RT of lexical-decision responses was computed for the correct trials in each condition. These data were subjected to a two-way repeated-measures analysis of variance, with relatedness (related or unrelated pairs) and practice (first or second half of the session) as within-subjects variables.

Only practice was significant $[\mathrm{F}(1,9)=8.85, \mathrm{p}<.025]$. Collapsed across the relatedness factor, lexical decisions were faster in the second half of the session than in the first by $78 \mathrm{msec}$. Mean RTs as a function of practice and relatedness are presented in Table 2 . An analysis of the data of individual subjects revealed that in both halves of the session, five subjects showed the relatedness effect and five did not.

The results of Experiment 2 suggest that repetition of this task may facilitate performance in general but does not influence the relatedness effect. Thus, the interaction of relatedness and task order that was found in Experiment 1 does not appear to have been due to the repetition of stimulus materials. As in the prime letter-search condition there, no effect of semantic relatedness was found in the present experiment.

\section{Discussion}

The commonly held notion of spreading activation encompasses the following assumptions: the spread of activation between concepts is automatic, the degree of activation is proportional to the semantic association between the concepts, and activation flows unidirectionally from the prime to the target. With respect to automaticity, Fischler (1977) showed that the occurrence of the context effect in a lexical-decision task does not depend upon an expectation of associated

Table 2

Mean Reaction Times (in Milliseconds) of Lexical Decision as a Function of Practice and Relatedness

\begin{tabular}{ccc} 
& \multicolumn{2}{c}{ Practice } \\
\cline { 2 - 3 } Relatedness & First Part & Second Part \\
\hline Related & 735 & 668 \\
Unrelated & 747 & 658 \\
\hline
\end{tabular}

pairs of words. However, there are some indications that the effect can be influenced by expectations, which are manipulated via changes in the proportion of related pairs within the entire stimulus set (Koriat, 1981; Tweedy \& Lapinski, 1981; Tweedy, Lapinski, \& Schvaneveldt, 1977). These findings are in line with the suggestion (Posner \& Snyder, 1975a, 1975b; Tweedy \& Lapinski, 1981) that the association effect has both automatic and attentional components. In our design, $50 \%$ of the word-word pairs were related. This percentage is higher than the percentage of related pairs in the low-validity condition used by Koriat (1981) and lower than his high-validity condition (20\% and $80 \%$ related pairs, respectively). Thus, our design should capture both attentional and automatic components. The fact that we have found a context effect in the prime naming task argues that it was not reduced expectations for related pairs that eliminated the effect in the prime letter-search task. It was the way the prime was processed that mattered.

What are the characteristics of processing the prime that produce a context effect? For the moment, we cannot convey the details of the "appropriate" processing mode. We can only point to Smith's (1979) and our idea that the necessary feature is that the prime be processed for meaning rather than probed for its constituents. Presumably, the extraction and use of meaning are necessary for activation to spread in the semantic network. This conclusion rests on the assumption that the facilitation of lexical decisions in the priming paradigm is a valid measure of the spread of activation in the semantic network. Although widely held, this assumption was recently challenged by Koriat (1981).

Koriat (1981) used a sequential priming paradigm and asked subjects to perform a lexical decision on the targets. He manipulated prime-target relatedness and associative strength. He found a relatedness effect that accorded with the notion of activation spread. However, the size of the relatedness effect was not dependent upon prime-target associative strength. This result is at odds with the idea that the context effect should reflect the degree of semantic association between the concepts involved. In addition, Koriat found that the amount of priming in lexical decisions is related to prime-target associations in both directions. This result does not fit with the expectation that "forward" links (prime to target) rather than "backward" links (target to prime) determine the degree of activation.

The concept of spreading activation was also invoked with regard to the Stroop effect reported by Warren $(1972,1974)$. Warren presented subjects with an auditory prime followed by a visual word printed in colored ink. The subjects were asked to name the color of the ink. The time for naming the ink color was longer when the visual word and the prime were semantically related than when they were not. Moreover, the time for naming the ink color increased with the strength of the 
forward association between the auditory word and the visual word. Backward associations had no effect. These results have been interpreted (Posner, 1978; Posner \& Snyder, 1975a, 1975b) as reflecting the activation of the visual word by the related auditory prime. This activation, in turn, produces interference with reporting the ink-color name.

The difference between Koriat's (1981) and Warren's $(1972,1974)$ results is puzzling, particularly in view of the fact that, for both, semantic distance was defined in terms of the same measure, namely, associative strength. Thus, contrary to the usual assumption that context effects in both color naming and lexical decision rely on spreading activation, it is possible that a different mechanism underlies the effect in each case. In the light of this conclusion and the difficulty raised for the appropriateness of the lexical-decision task as a tool for measuring activation spreading from the prime, one question of interest is whether the pattern of results found in Experiments 1 and 2 with lexical decision would be produced when a color-naming task was used. If interference with color naming were found when primes are named but not when primes are searched for letters, the conclusion that relatedness effects depend upon the mode of processing of the prime would be strengthened. Such a result would support the generality of this claim, as well as provide further evidence on the issue of whether lexical-decision and colornaming context effects are mediated by the same mechanism. The following experiment investigated these issues.

\section{EXPERIMENT 3}

In the present experiment, we used the same prime tasks that had been used in Experiment 1. Each subject searched the prime for a letter on half of the trials and named the prime on the other half. The target task was changed from a lexical-decision to a color-naming task. The subjects were asked to name the color of the targets as quickly as possible. In the lexical-decision task, priming was expected to have a facilitatory effect on target RTs; in the color-naming situation, an inhibitory effect was expected. The assumed spread of activation from the prime to a related target would be expected to facilitate the target's processing rate. Thus, the word name would be delivered more quickly to the output mechanisms, producing response competition and resulting in more interference with reporting the color name than when prime-target pairs are unrelated (Keele, 1973; Posner, 1978). In the two prime tasks used here, we expected to find interference when the prime was named and no, or at least less, interference when the prime was searched for a letter.

\section{Method}

In general, the method was similar to that of Experiment 1. The departures from the previous experimental method are presented here.
Stimuli and Apparatus. The stimulus list from Experiment 1 was used, with the following changes. Target letter strings were colored and could appear in one of four different colors: orange, green, blue, or purple. Each color was equally probable within each condition, and the allocation of colors to words was random, with the constraint that no color could appear more than three times in a row.

The apparatus was the same as in Experiment 1. From the original stimulus list, we eliminated trials that included color words and thus produced a total of 144 experimental and 42 practice trials.

Procedure. Each subject participated in a 1-h session. The session consisted of two parts that differed in the task applied to the prime. In one part, the subjects were asked to name the prime, and in the other, they were asked to search it for a letter. In contrast with Experiment 1, the response to the prime letter search was manual rather than vocal.

For the target words, the subjects were asked to name the color and to ignore the meaning of the words. The target task remained the same throughout the session. Vocal RTs for color naming were recorded by the voice-operated relay used in Experiment 1 . The correctness of the color responses was typed into the computer by the experimenter.

Subjects. Twenty University of Oregon students were recruited either from the subject pool of the Cognitive Laboratory or from psychology classes. They were paid $\$ 3.00$ or received class credit, respectively, for their participation. All subjects were native English speakers, and none had participated in any of the previous experiments.

\section{Results}

For each subject, a median RT was computed for correct color-naming respons in each condition. These medians were subjected to a three-way repeated-measures analysis of variance with prime task (naming or letter search) and semantic relatedness (related or unrelated pairs) as within-subjects factors and order (prime letter search first or prime letter search second) as a betweensubjects factor.

Only the semantic relatedness factor was significant $[F(1,18)=6.76, p<.025]$. Collapsed across all other factors, color responses to related words were slower by $19 \mathrm{msec}$ than to unrelated words. Mean RTs as a function of semantic relatedness and prime task are presented in Table 3. An analysis of the data of individual subjects revealed that in the naming task, 15 subjects showed the relatedness effect and 5 did not $(p<.021$ in the sign test); when the primes were searched for letters, 10 subjects showed the effect and 10 did not.

\section{Discussion}

In contrast with Experiment 1, the color-naming task produced a main effect of relatedness that was not qualified by an interaction with the prime task. Al-

Table 3

Mean Reaction Times (in Milliseconds) of Color Naming as a Function of Relatedness and Prime Task

\begin{tabular}{ccc}
\multicolumn{3}{c}{ Function of Relatedness and Prime Task } \\
\hline & \multicolumn{2}{c}{ Prime Task } \\
\cline { 2 - 3 } Relatedness & Letter Search & Naming \\
\hline Related & 812 & 794 \\
Unrelated & 799 & 769 \\
\hline
\end{tabular}


though the relatedness $x$ prime task interaction did not reach significance, the pattern of results was consistent with that of Experiment 1. That is, the context effect was larger when the prime was named than when it was searched for a letter. The inhibitory effect that appeared when the prime was named is not new, having been reported by Warren $(1972,1974)$. The small effect that appears in the prime letter-search condition is at odds with our previous results. Before reaching any firm conclusion with respect to this effect, we tried to replicate it in the next experiment.

\section{EXPERIMENT 4}

This experiment is a replication of the prime lettersearch condition of the previous experiment. On half of each session, we used a literal replication of that condition. On the other half, we administered a change in the prime response mode. That is, instead of a manual response to the prime, the subjects were asked to respond vocally ("yes" or "no"). The use of two response modes will enable us to claim generality for the effect.

\begin{abstract}
Method
The present experiment was similar to the prime lettersearch condition of Experiment 3, with the following exceptions. Each subject participated in the prime letter-search task twice. In one part of the experiment, the subjects responded to the prime vocally; in the other part, they responded manually. Throughout the experiment, the subjects named the color of the targets, as in the previous experiment. The manual-prime condition (manual response to the prime and vocal response to the target) was an exact replication of the letter-search condition in Experiment 3, and the vocal-prime condition was a replication in which the response mode to the prime was changed.

Subjects. Fourteen University of Oregon students were recruited from either the subject pool of the Cognitive Laboratory or psychology classes. They were paid $\$ 3.00$ or received class credit, respectively, for their participation. All subjects were native English speakers, and none had participated in any of the previous experiments.
\end{abstract}

\section{Results}

For each subject, a median RT was computed for correct color-naming trials in each condition. These medians were subjected to a three-way repeated-measures analysis of variance, with prime response mode (vocal or manual) and semantic relatedness (related or unrelated) as within-subjects factors and order (vocal prime first or vocal prime second) as a between-subjects factor.

Only two effects were significant. When subjects responded to the prime vocally, their color-naming responses to the targets were faster by $32 \mathrm{msec}$ than when they responded to the prime manually $[F(1,12)=$ $9.53, \mathrm{p}<.01]$. However, this factor interacted with order $[F(1,12)=19.96, p<.001]$. When the prime was responded to vocally in the first part of the experiment, there was a small advantage $(14 \mathrm{msec})$ of the manualprime over the vocal-prime condition. When the subjects responded vocally to the prime in the second part of
Table 4

Mean Reaction Times (in Milliseconds) of Color Responses as a Function of Relatedness and Mode of Prime Response

\begin{tabular}{ccc}
\hline & \multicolumn{2}{c}{ Mode of Prime Response } \\
\cline { 2 - 3 } Relatedness & Vocal & Manual \\
\hline Related & 804 & 834 \\
Unrelated & 794 & 829 \\
\hline
\end{tabular}

the experiment, the reverse pattern appeared (they were faster by $79 \mathrm{msec}$ in the vocal-prime condition than in the manual-prime condition). The relatedness factor did not produce a significant main effect and did not interact with other factors. The data of the individual subjects revealed that: in the vocal-prime condition, eight subjects showed a relatedness effect and six did not; in the manual-prime condition, five showed the effect and seven did not; and two had the same RT in the related and unrelated pairs.

The most important result of the present experiment was that neither a main effect of relatedness nor an interaction of it with prime response mode occurred. The data relevant to this issue are presented in Table 4. The net interference due to relatedness in the two prime response conditions was quite small and insignificant.

\section{GENERAL DISCUSSION}

In the foregoing experiments, we used two different tasks in order to observe the effects of priming. One task was lexical decision, and the other was color naming. It is interesting to note that the two tasks gave rise to different manifestations of priming effects. In the lexical decision task, semantic priming led to facilitation of response, whereas in color naming, priming led to inhibition of responses. As we noted earlier, these two results have been reported separately elsewhere. It is important to show that one can observe the two patterns when using the same set of stimuli and general design. Our finding supports the claim that, in general, the underlying mechanism for the two opposite patterns is the same. The spread of activation from the prime concept to related nodes in semantic memory facilitates activation of a semantically related target. This facilitation is beneficial in some circumstances, as when a decision with respect to the lexical status of the target has to be made. In other circumstances, it may be detrimental, as when the response involves a dimension that is not related to the target's lexical status (i.e., its color) but that can be interfered with by the word name.

Two tasks were applied to the prime. One was naming, and the other was letter search. When the prime is searched for a letter, its effect on the target is either eliminated completely or is reduced to an insignificant level. It is reasonable to conclude that the manipulation of prime processing results in a major change in the effect on the 
target. It should be noted that, in some cases, the pattern of means suggests that the priming effect may be substantially reduced but not completely eliminated under the letter-search condition. It is possible that more sensitive procedures might demonstrate a small but reliable priming effect when a letter-search priming task is used. The results most favorable for such an idea appeared in Experiment 3. However, the individual subject data indicate that just as many subjects produced a negative priming effect as showed a positive priming effect. This suggests to us that, even with a more sensitive measure, it might be difficult to find a priming effect that is reliable across subjects.

This effect is quite different from the effects of expectancy discussed by Becker (1980) and Tweedy and Lapinski (1981). Tweedy and Lapinski stressed the importance of the proportion of trials consisting of related pairs and the order of presentation of the lists with the various proportions. Becker discussed the effect of the composition of the list on the range of potential targets. In the present set of experiments, these two factors were held constant. Thus, neither kind of expectancy set is very likely to have affected our results. However, there is another kind of expectancy that may be generated here. This is related to the role of meaning in the processing of targets. It is possible that a letter-search task in some way reduces the expectation that semantically related pairs will occur. This possibility is unlikely because in our experiments, each subject was presented with the stimulus material twice, once with each task. Had this possibility been a factor, one would expect a three-way interaction of relatedness, prime task, and order in Experiments 1 and 3 . This was not the case.

We suggest that the present results can be explained by the operation of the attentional system. Several studies suggest that attention may select a particular meaning according to context, which may then affect the pattern of activation that occurs in the semantic network. Schvaneveldt, Meyer, and Becker (1976) presented subjects with three-word sequences. The three words were presented successively, and the subjects were asked to make a lexical decision for each word. The middle word was polysemous. The other two words could be related to the same meaning of the polysemous word (e.g., HAND-PALM-WRIST), the congruent case, or to different meanings (TREE-PALMWRIST), the incongruent case. In the control, or unbiased, case, the polysemous word was preceded by an unrelated word (SPEED-PALM-WRIST). The lexicaldecision response for the third word was facilitated in the congruent case. The facilitation occurred to a lesser degree in the unbiased case and not at all in the incongruent condition. Thus, it seems that the context presented by the first word focused attention on one meaning of the polysemous word and prevented facilitation of the other meaning.
Marcel (1980) used a similar paradigm to produce an even more dramatic effect. He presented the three words successively and, in one condition pattern masked the polysemous word. The use of the pattern mask created a condition in which the subjects were unaware of the presence of the polysemous word. When the pattern mask was used, both meanings of the polysemous word were activated, producing a facilitation effect in both the congruent and incongruent conditions. However, in the no-mask condition, facilitation appeared only for words related to the biased meaning of the polysemous word (i.e., the congruent case). Marcel's no-mask condition essentially replicated Schvaneveldt et al.'s (1976) findings. It seems that when subjects were aware of the polysemous word (no-mask condition), they selected only one of its interpretations, according to the context.

In our study, it is possible that when the prime was searched for a letter, attention was drawn to the letter level, and as a result, no attention was allocated to the semantics of the prime words. This reduced the priming effect in the subsequent lexical-decision task. When the primes were named, attention was allocated to the meaning of the prime, which caused activation of its node in the semantic network and a spread of activation to related concepts. This spread of activation gave rise to facilitation of lexical decisions and inhibition of color naming. The withdrawal of attention from the semantics in the letter-search task may affect performance in two different ways. It may inhibit lexical activation and/or the spread of activation and thus prevent the usual priming effect from occurring. On the other hand, lexical activation and/or the spread of activation may persist, but the altered attentional focus may result in a decay of this activation, which prevents it from affecting the subsequent target. The present set of experiments does not allow for a choice between these two alternatives, and further research is needed for this end. Whichever alternative is correct, our results support the notion that it is possible to eliminate the effects of spreading activation when attention is drawn to processing the prime in a nonsemantic fashion.

\section{REFERENCES}

Becker, C. A. Semantic context effects in visual word recognition: An analysis of semantic strategies. Memory Cognition, $1980,8,493-512$.

Collins, A. M., \& Lortus, E. F. A spreading-activation theory of semantic processing. Psychological Review, 1975, 82, 407-428.

Fischler, I. Associative facilitation without expectancy in a lexical decision task. Journal of Experimental Psychology: Human Perception and Performance, 1977, 3, 8-26.

KeEle, S. W. Attention and human performance. Pacific Palisades, Calif: Goodyear, 1973.

Keppel, G., \& Strand, B. Z. Free association responses to the primary responses and other responses selected from the Palermo-Jenkins norms. In L. Postman \& G. Keppel (Eds.), Norms of word association. New York: Academic Press, 1970.

Korist, A. Semantic facilitation in lexical decision as a function 
of prime-target association. Memory \& Cognition, 1981, 9, $587-598$.

Krueger, L. E., \& Weiss, M. E. Letter search through words and nonwords: The effect of fixed, absent or mutilated targets. Memory \& Cognition, 1976, 4, 200-206.

MARCEL, A. J. Conscious and preconscious recognition of polysemous words: Locating the selective effects of prior verbal context. In R. S. Nickerson (Ed.), Attention and performance VIII. Hillsdale, N.J: Erlbaum, 1980.

Meyer, D. E., \& Schvaneveldt, R. W. Facilitation in recognizing pairs of words: Evidence of a dependence between retrieval operations. Journal of Experimental Psychology, 1971, 90, 227-243.

Meyer, D. E., \& Schvaneveldt, R. W. Meaning, memory structure and mental processes. Science, 1976, 192, 23-27.

NeELy, J. H. Semantic priming and retrieval from lexical memory: Evidence for facilitatory and inhibitory processes. Memory \& Cognition, 1976, 4, 648-654.

NeELY, J. H. Semantic priming and retrieval from lexical memory: Roles of inhibitionless spreading activation and limitedcapacity attention. Journal of Experimental Psychology: Gen. eral, 1977, 106, 226-254.

Posner, M. I. Chronometric explorations of mind. Hillsdale, N.J: Erlbaum, 1978.

Posner, M. I., \& Snyder, C. R. R. Attention and cognitive control. In R. L. Solso (Ed.), Information processing and cognition. Hillsdale, N.J: Erlbaum, 1975. (a)

Posner, M. I., \& SNyder, C. R. R. Facilitation and inhibition in the processing of signals. In P. M. A. Rabbitt (Ed.), Attention and performance $V$. London: Academic Press, 1975. (b)
Postman, L. The California norms: Association as a function of word frequency. In L. Postman \& G. Keppel (Eds.), Norms of word association. New York: Academic Press, 1970.

Schvaneveldt, R. W., \& McDonald, J. E. Semantic context and the encoding of words: Evidence for two modes of stimulus analysis. Journal of Experimental Psychology: Human Perception and Performance, 1981, 7, 673-687.

Schvaneveldt, R. W., Meyer, D. E., \& Becker, C. A. Lexical ambiguity, semantic context, and visual word recognition. Journal of Experimental Psychology: Human Perception and Performance, 1976, 2, 243-256.

Sм гтн, M. C. Contextual facilitation in a letter search task depends on how the prime is processed. Journal of Experimental Psychology: Human Perception and Performance, 1979, 5, 239-251.

Tweedy, J. R., \& Lapinski, R. H. Facilitation word recognition: Evidence of strategic and automatic factors. Quarterly Journal of Experimental Psychology, 1981, 33A, 51-59.

Tweedy, J. R., Lapinski, R. H., \& Schvaneveldt, R. W. Semantic context effects on word recognition: Influence of varying the proportion of items presented in an appropriate semantic context. Memory \& Cognition, 1977, 5, 84-89.

W ARrEN, R. E. Stimulus encoding and memory. Journal of Experimental Psychology, 1972, 94, 90-100.

WARREN, R. E. Association, directionality, and stimulus encoding. Journal of Experimental Psychology, 1974, 102, 151-158.

(Manuscript received November 5, 1982; revision accepted for publication April 19, 1983.) 